\title{
Comparative Analyses of Chloroplast Genomes from Six Rhodiola Species: Variable DNA Markers Identification and Phylogenetic Inferences
}

\section{Kaihui Zhao}

Tibet Agriculture and Animal Husbandry University

\section{Lianqiang Li}

Tibet Agriculture and Animal Husbandry University

\section{Hong Quan}

Tibet Agriculture and Animal Husbandry University

Junbo Yang

Chinese Academy of Sciences

\section{Zhirong Zhang}

Chinese Academy of Sciences

\section{Zhihua Liao}

Tibet Agriculture and Animal Husbandry University

\section{Xiaozhong Lan ( $\square$ lanxiaozhong@163.com )}

Tibet Agriculture and Animal Husbandry University

\section{Research Article}

Keywords: Rhodiola, chloroplast genome, divergent hotspots, phylogenetic analysis

Posted Date: January 24th, 2022

DOI: https://doi.org/10.21203/rs.3.rs-1145757/v1

License: (9) This work is licensed under a Creative Commons Attribution 4.0 International License. Read Full License 


\section{Abstract \\ Background}

As a valuable medicinal plant, Rhodiola has a very long history of folk medicine used as an important adaptogen, tonic, and hemostatic. However, our knowledge of the chloroplast genome level of Rhodiola is limited.

\section{Results}

Six Rhodiola complete chloroplast genomes were determined and compared to another Rhodiola cp genome at the genome scale. The results revealed a cp genome with a typical quadripartite and circular structure that ranged in size from 150,771 to 151,891 base pairs. These Rhodiola cp genomes contain genome organization, gene number, gene order, and GC content that are similar to many angiosperm $\mathrm{cp}$ genomes. Repeats and microsatellites were detected. Thirteen mutational hotspots for genome divergence were determined and could be used as candidate markers for phylogenetic analyses and Rhodiola species identification. The phylogenetic relationships inferred by members of Rhodiola cluster into two clades: dioecious and hermaphrodite. Our findings are helpful for understanding Rhodiola's taxonomic, phylogenetic, and evolutionary relationships.

\section{Conclusions}

Comparative analysis of chloroplast genomes of Rhodiola facilitates medicinal resource conservation, phylogenetic reconstruction and biogeographical research of Rhodiola.

\section{Introduction}

As traditional natural plant pharmaceuticals and health food, Rhodiola is belonging to the family of Crassulaceae and mainly distributed in alpine regions of Asia and Europe [1-3]. 73 species of Rhodiola plants are distributed in China, and the Qinghai-Tibet Plateau has the most species [4]. The extract of $R$ hodiola plants, especially $R$. crenulata and $R$. rosea, has various pharmacological effects such as antihypoxia, fatigue, tumors, radiation, aging, and improvement of mental and physical functions [5]. With the in-depth research on the pharmacological effects of Rhodiola plants, the demand for Rhodiola resources is increasing. Due to the fragile ecological environment of the Qinghai-Tibet Plateau and the lack of artificial cultivation techniques, relying solely on the digging of wild resources can easily lead to the reduction of Rhodiola plant resources and the loss of genetic diversity resources [6].

Due to the variety of Rhodiola plants, the source of commercial medicine of Rhodiola is very complicated, but the pharmacodynamics of different species of Rhodiola have a significant difference in clinical efficacy. The traits of the medicinal plants of Rhodiola and the characteristics of the microstructure are 
similar [7]. At present, $R$. crenulata is the only primitive plant of the Rhodiola medicinal herbs contained in the Chinese Pharmacopoeia (2020 Edition). The development and research of its alternative varieties is imminent. In recent years, the mixed use of plant roots and rhizomes of distinct species of Rhodiola has occurred frequently. Researchers have studied the Rhodiola Herbal Slices on the market using DNA barcoding technology, only $40 \%$ of the samples are $R$. crenulata collected in the Chinese Pharmacopoeia [8]. The chaotic use of Rhodiola medicinal materials directly affects the safety and efficacy of clinical medications, and coupled with unrestricted collection, the number of wild resources has decreased dramatically. Therefore, in order to realize the protection and sustainable development of Rhodiola plants, it is necessary to conduct in-depth research on the identification and genetic diversity of their species.

To solve the problem of Rhodiola plant identification, Wang et al. developed random amplification polymorphic DNA (RAPD) and inter-simple sequence repeat (ISSR) primers to identify $R$. angusta, $R$. crenulata, $R$. bupleuroides, and $R$. sachalinensis [9]. Li et al established a method for classifying and identifying $R$. quadrifida and $R$. crenulata based on nuclear magnetic resonance $1 \mathrm{H}-\mathrm{NMR}$ fingerprintschemical pattern recognition technique [10]. Zhu et al. found that the internal transcribed spacer 2 (ITS2) sequence can effectively distinguish $R$. crenulata and $R$. rosea [11]. Booker et al. used nuclear magnetic resonance spectroscopy coupled with high performance thin layer chromatography techniques to comprehensively analyze $R$. crenulata and $R$. rosea collected in markets around the world [12]. These advanced identification methods currently used can solve the identification problems of some Rhodiola plants, but they also have the disadvantages of having a narrow application range and high identification cost.

DNA barcoding is a new species identification technology developed in recent years [13]. It eliminates the obstacles of traditional morphological recognition methods that rely on long-term experience. The plant chloroplast (cp) genome, as a research hotspot for screening DNA barcoding sequences, can also be used as a super-barcoding for phylogenetic and species identification studies [14]. The use of the $\mathrm{cp}$ genome to solve the problem of difficult classification of related species is of great significance for species identification in herbal medicine and even the entire plant community. Chen et al. proposed that using the whole genome as a super-barcode can effectively identify Ligularia plants [15]. Zhong et al. found that 41 Dendrobium species can be effectively identified based on the whole cp genome, and Dendrobium officinale from 3 different places of production can also be distinguished [16]

In this study, we sequenced the cp genomes of $R$. tangutica, $R$. wallichiana, $R$. quadrifida, $R$. bupleuroides, $R$. gelida, and $R$. henryi using Illumina technology followed by reference-guided assembly of de novo contigs. Our aims were: 1 ) to detect the variations of long repeats and SSRs in 6 Rhodiola cp genomes; 2) to identify divergence hotspots as potential genetic markers for Rhodiola DNA barcoding; and 3) to construct a phylogeny for Rhodiola species using protein coding sequences of the cp genome and infer their phylogenetic location within Crassulaceae.

\section{Results}




\subsection{General Features of the Six Rhodiola Cp Genomes}

The cp genomes of $R$. tangutica (2.4Gb), R. wallichiana (2.1Gb), R. quadrifida (2.4Gb), R. bupleuroides (2.2Gb), $R$. gelida $(2.1 \mathrm{~Gb})$, and $R$. henryi $(2.3 \mathrm{~Gb})$ were sequenced with approximately $2.0 \mathrm{~Gb}$ of paired-end reads, respectively. Clean reads were achieved by removing adaptors and low-quality read pairs. The recovered clean reads for $R$. quadrifida, $R$. tangutica, $R$. wallichiana, $R$. bupleuroides, $R$. gelida, and $R$. henryi were $1,737,149,1,013,832,839,613,973,418,866,547$, and $1,092,141$, respectively (Table S1). Six Rhodiola complete cp genome maps (Figure 1) were obtained through de novo genome sequencing and assembly with the reference $R$. rosea (MH410216) genome. The average organelle coverage for $R$. quadrifida, $R$. tangutica, $R$. wallichiana, $R$. bupleuroides, $R$. gelida, and $R$. henryi with the reference genome reached 1,378, 262, 254, 193, 203, and 313, respectively (Table S1).

The cp genome size ranged from $150,771 \mathrm{bp}$ in $R$. quadrifida to $151,891 \mathrm{bp}$ in $R$. henryi, which included $82,211 \mathrm{bp}$ ( $R$. tangutica) to 83,095 bp ( $R$. gelida) long single copy (LSC) regions, and 16,991bp ( $R$. quadrifida) to $17,104 \mathrm{bp}$ ( $R$. tangutica) short single copy (SSC) regions, and separated by a pair of 25,773 bp (R. quadrifida) to 25,887 bp ( $R$. henryi) inverted repeat (IR) regions (Fig. 1; Table S1). There were 85

protein-coding genes, 37 tRNA genes, and 8 rRNA genes that were identified in each Rhodiola cp genome (Table S2). Among these unique genes, 15 genes harbored one intron and three genes $(y c f 3, c / p P$, and rps12) harbored two introns (Table S2).

\subsection{Long repeats and SSRS}

Repeat sequences have been applied extensively for phylogeny, population genetics, genetic mapping, and forensic studies [17]. We used REPuter to identify repeat sequences in the cp genomes of 6 Rhodiola species. A total of 144 pairs of repeats were detected in the 6 Rhodiola cp genomes, with the repeat length range from 30 to $62 \mathrm{bp}$ (Fig. 2A). The cp genomes of 6 Rhodiola have 5, 15, 8, 11, 12 and 9 forward repeats and 10,14, 13, 16, 13 and 14 palindromic repeats (Fig. 2B). Reverse repeats and complementary repeats only exist in the cp genome of R. gelida (Fig. 2B). The long repeat lengths of 30, $31,32,40$, and 41 bp existed in all 6 Rhodiola cp genomes (Fig. 2A). Long repeat lengths of 33, 34, and 36 bp were found the least often and only existed in the $R$. gelida and $R$. bupleuroides $\mathrm{cp}$ genomes, respectively (Fig. 2A).

Simple sequence repeats (SSRs) are usually 1-6 bp tandem repeat DNA sequences and are widely used as molecular markers with their polymorphic to identify closely related species [18]. In our study, SSRs in 6 Rhodiola cp genomes were identified using MISA software. The number of SSRs in the 6 Rhodiola species ranged from 186 (R. wallichiana) to 200 (R. gelida) (Fig. 3A). The numbers and distribution of all SSR types were similar and conserved in the 6 Rhodiola cp genomes, except for pentanucleotide, which only existed in $R$. bupleuroides (Fig. 3B). Mononucleotide repeat motifs were occupied the largest proportion in these SSRs, ranging from $63 \%$ ( $R$. bupleuroides) to $65 \%(R$. henryi). No hexanucleotide repeat motifs were found in the 6 Rhodiola cp genomes (Fig. 3B). Among these mononucleotide repeats, $A$ and $T$ nucleotides were the most common, while tandem $G$ or $C$ repeats were quite rare (Fig. $3 \mathrm{~A}$ ), which was in concordance with the other research results $[19,20]$. These SSR markers could be used to examine 
the genetic structure, differentiation, diversity, and maternity in the 6 Rhodiola species and their relative species in future studies.

\subsection{Divergence Hotspots}

We selected eight Rhodiola cp genome sequences to be compared and plotted using the mVISTA software with the annotated cp genome of $R$. rosea as a reference to elucidate the level of sequence divergence (Fig. 4). Based on the overall sequence identity, the results indicated that the coding regions exhibit lower divergence levels than the non-coding regions and two IR regions exhibit higher conservation than the remaining sequences across the whole chloroplast genome, as can be seen in other plants [21, 22]. Furthermore, the results showed that the $y c f 1$ and $t r n H-G U G-p s b A$ sequences of Rhodiola were highly divergent regions.

In addition, the nucleotide diversity $(\mathrm{Pi})$ values were calculated to evaluate the sequence divergence among 22 Rhodiola cp genomes (Table S3). The genetic distance of all 75 protein-coding genes varied from 0 to 0.01143 (ycf1) with an average value of 0.00444 (Figure 5A). Based on a considerably higher $\mathrm{Pi}$ value of $>0.009$, we found seven highly variable regions ( $y c f 1, r p s 15, n d h F, r p o C 1, r p s 8, r p / 20, r p s 18$, and matK) (Fig. 5A). These values of the non-coding regions ranged from 0 to 0.04122 (trnH-GUG-psbA) with an average value of 0.01057 (Fig. 5B). A total of five mutational hotspots that showed high values of PI ( $\geq 0.02$ ) were identified, including trnH-GUG-psbA, rps15-ycf1, trnG-GCC-trnR-UCU, trnC-GCA-petN, and $n d h F-r p / 32$. The analysis revealed that the protein-coding regions exhibit lower divergence levels than non-coding regions. These hotspot regions could be utilized as potential molecular markers for phylogenetic studies and the identification of Rhodiola species.

\subsection{Phylogenetic Analysis Within Rhodiola}

Complete cp genomes comprise abundant phylogenetic information, which could be applied to evolution and phylogenetic studies of angiosperms because of several advantages, such as high accuracy and resolution [23]. In order to clarify the phylogenetic position of six newly assembled Rhodiola within the Saxifragales, 38 protein-coding genes from 55 available complete cp genome sequences in Saxifragales were used for phylogenetic analyses. Maximum likelihood (MI) and Bayesian inference (BI) were used to construct phylogenetic tree with Rosa rugosa as an outgroup. The phylogenetic tree showed that all Rhodiola species formed a monophyletic clade and then were classified into two separate branches (Fig. 6), which is inconsistent with the record in Flora of China [1]. The dioecious clade composed of nine dioecious Rhodiola species, and the hermaphrodite clade included all the hermaphrodite species.

\section{Materials And Methods}

\subsection{Plant Materials and DNA Extraction}


Plant materials of Rhodiola were collected in Nyingchi (Tibet, China). The specimens of Rhodiola have been kept at the Tibet Agriculture \& Animal Husbandry University. Total genomic DNA was extracted from silica-gel-dried leaves using the modified CTAB method [24]. The quantity and quality of extracted genomic DNA were determined by gel electrophoresis and NanoDrop 2000 Spectrophotometer (Thermo Scientific, Carlsbad, CA, USA).

\subsection{Chloroplast Genome Sequencing, Assembly and Annotation}

Genomic DNA was randomly fragmented using physical methods. Paired-end sequencing libraries were constructed according to the Illumina standard protocol (Illumina, San Diego, CA, USA). Sample sequencing was carried out on an Illumina Hiseq X-Ten platform. Total genomic DNAs were also sent to BGI (Shenzhen, China) for library (400 bp) preparation for genome skimming sequencing. Paired-end (150 bp) sequencing was conducted on the Illumina HiSeq X-10 platform, generating $\sim 2 \mathrm{~Gb}$ data per sample. Raw reads were filtered by quality control software NGS QC Toolkit v2.3.333 to obtain high quality Illumina data [25].

Next, filtered reads were de novo assembled using NOVOPlasty [26] with parameters of K-mer (33). These assembled chloroplast genomes were annotated in GeSeq [27], coupled with manually edited start and stop codons in Geneious 11.1.4 [28] (Biomatters Ltd., Auckland, New Zealand) with a reference R. rosea chloroplast genome (Genbank accession number MH410216). In addition, all tRNA genes were further verified using tRNAscan-SE v1.21 [29]. The border region between the inverted repeat (IR) and the large single copy (LSC), also between inverted repeats and small single copy (SSC) junction were determined through local BLAST software. Finally, the circular gene maps of Rhodiola plastomes were drawn utilizing the Organellar Genome DRAW tool (OGDRAW) [30].

\subsection{Comparative Genomic Analysis and Molecular Marker Identification}

To detect variations within Rhodiola cp genomes, we compared the cp genomes of $R$. crenulate, $R$. rosea, and the six newly assembled Rhodiola cp genomes by mVISTA [31]. The nucleotide diversity of the Rhodiola cp genomes was detected by DNA Sequence Polymorphism (DnaSP) software [32].

\subsection{Characterization of Repeat Sequence and SSRs}

The long repetitive sequences were detected using REPuter with a $30 \mathrm{bp}$ minimum repeat size and a Hamming distance of 3 [33]. Simple sequence repeats (SSRs) in the cp genomes were identified via the MISA perl script [34] with the minimum number of repeats set to 8, 5, 3, 3, 3 and 3 for mono-, di-, tri-, tetra-, penta-, and hexa-nucleotides, respectively.

\subsection{Phylogenetic Analysis}

The phylogenetic analysis was performed on six newly assembled Rhodiola cp genomes, another 55 Saxifragales species, and one outgroup Rosa rugosa, all of which were down loaded from the NCBI 
(Table S4) except those of six newly assembled Rhodiola cp genomes. Molecular phylogenetic trees, using aligned sequences of 38 protein-coding genes (Table S5) with MAFFT 7.0 [35] and adjusted manually where necessary, were constructed using IQ-TREE (Nguyen et al.,2015) and MrBayes 3.2.6 software [36] under the GTRGAMMA model.

\section{Discussion}

Six Rhodiola cp genomes were sequenced and assembled in our study, and this information was used to identify candidate DNA markers and infer Rhodiola phylogeny. The size of the cp genome, the length of the SSC, LSC, and IR regions, the content of the GC, and gene content demonstrated a high degree of similarity among the genomes, implying that Rhodiola species shared low diversity [4]. The IR region, however, has a larger GC content than the LSC and SSC regions. In most angiosperm chloroplasts, there are 74 protein-coding genes, with an additional five in a few species [37]. Six newly assembled Rhodiola cp genomes contain 85 protein-coding genes, 37 tRNA genes, and 8 rRNA genes, which is consistent with previous studies [4].

The whole cp genome contains abundant mutation sites, which can be used directly as a super barcode for species identification. As with hypervariable regions of the genome, they can also be screened out as potential molecular markers [38, 39]. At present, many species have been successfully identified based on the chloroplast genome, especially the species with frequent hybridization and apomixis [39-41]. Ycf1, $r p s 15, n d h F, r p o C 1, r p s 8, r p / 20, r p s 18$ and matK genes in CDS showed significant variation and high sequence variations were found in intergenic regions as follows: trnH-GUG-psbA, rps15-ycf1, trnG-GCCtrnR-UCU, trnC-GCA-petN, and ndhF-rp/32 (Figure 6). These regions can also be used as candidate markers for elucidating the phylogenetic relationship among Rhodiola species. In many species, $\mathrm{TrnH}$ GUG-psbA and matK are the most mutated hotspots for species identification, such as Kengyilia [42], Apocynaceae [43] and Orchidinae [44]. Ycf1 marker has good species identification resolution in Pinus at the within-genus level relationships [45]. Ycf1 has a better effect on the identification of Rhodiola due to its longer sequence ( 5800bp). We recommend that the ycf1 gene be used to reconstruct phylogenetic relationships of Rhodiola where there is a lack of genomic information.

Our phylogenetic analysis strongly supported the monophyly of Rhodiola species, which is consistent with previous studies $[4,46]$. All Rhodiola species are classified into two separate branches (dioecious and hermaphrodite), which supports the view of Zhao et al [4]. What interests us is that $R$. wallichiana was gathered in the hermaphrodite clade, and we think that we may have selected a rare unisexualis among $R$. wallichiana. So, we speculate that unisexual $R$. wallichiana and bisexual $R$. wallichiana may have huge genetic differences. In addition, the dioecious clade also contains $R$. integrifolia, which has been temporarily classified as a hybrid between $R$. rosea and $R$. rhodantha [47]. Our phylogenetic analyses also revealed that there are close relationships between Crassulaceae and Saxifragaceae, supporting the view that there is a common origin between them. In some traditional angiosperm classification systems, Saxifragaceae is the largest group of garbage bins in angiosperms, and many branches that are unrelated in evolution are forcibly pieced together into a highly multi-line group. Our 
phylogeny suggested that the Penthoraceae and Haloragidaceae were clustered into one clade, indicating their close relationship. The APGII research considers that Penthoraceae can be selectively combined with Haloragidaceae [48], our research seems to support this view. However, since there is only one chloroplast genome data in two families and lack of data on the cp genome of more species, we believe that when more species in the two families have been sequenced to accurately determine their evolutionary relationship.

\section{Conclusions}

In this study, we determined and characterized six cp genome sequences of Rhodiola, which are commonly used as Tibetan medicinal materials. The size of the genome, the structure and organization of genes were shown to be conservative, which is similar to those reported cp genomes of Rhodiola species. To develop molecular markers for future phylogeographic and population genetic studies, thirteen mutational hotspots were identified, including ycf1, rps15, ndhF, rpoC1, rps8, rp/20, rps18, matK, trnH-GUG-psbA, rps15-ycf1, trnG-GCC-trnR-UCU, trnC-GCA-petN, and ndhF-rp/32. The results of phylogenetic analysis showed that Rhodiola species were clustered into two clades: dioecious and hermaphrodite, with strong support values. The complete $\mathrm{cp}$ genome sequences that were newly assembled facilitate medicinal resource conservation, phylogenetic reconstruction, and biogeographical research of Rhodiola.

\section{Declarations}

Acknowledgements: We are grateful to all reviewers for their valuable comments on the manuscript.

Author Contributions: KZ and XL conceived and designed the work. HQ, ZZ, and JY collected the samples. KZ performed the experiments and analyzed the data; KZ and LL wrote the manuscript; ZL and XL revised the manuscript. All authors gave final approval of the paper.

Funding: This work was supported by the Tibet Autonomous Region Office and School Joint Foundation (Grant No. XZ202001ZR0038G), The National Natural Science Foundation of China (Grant No. 81660628) , The Special Support Plan for the Talent of Fifth WANREN Plan in China®Grant No.2020366囚,The Forth National Survey of Traditional Chinese Medicine Resources, Chinese or Tibet Medicinal Resources Investigation in Tibet Autonomous Region (State Administration of Chinese Traditional Medicine 20190411-542222), The Key R \& D program of Yunnan Province, China (grant no. 202103AC100003), The Large-scale Scientific Facilities of the Chinese Academy of Sciences (Grant No. 2017-LSF-GBOWS-02).

Availability of data and materials: The datasets supporting the results of this publication are included within the article and Additional files 1,2,3,4,5. The chloroplast genome data of $6 \mathrm{cp}$ genome sequences of Rhodiola used for analysis could be obtained from NCBI, and their accession numbers are as follow: $R$. wallichiana, OL742458; $R$. henryi, OL742459; $R$. gelida, OL742460; $R$. bupleuroides, OL742461; $R$. 
tangutica, OL742462; $R$. quadrifida, OL742463. Data for our newly assembled six Rhodiola chloroplast genomes are also available in Supplementary S6. Voucher specimens of 6 Rhodiola were deposited in the Kunming Institute of Botany, and their accession numbers are as follow: $R$. wallichiana, WH-2013-029; $R$. henryi, GanQL691; R. gelida, ChenSL1377; $R$. bupleuroides, LiuJQ-08XZ-062; $R$. tangutica, ChenSL0277; $R$. quadrifida, ChenSL0149.

\section{Ethics approval and consent to participate}

Not applicable. No specific permits were required for the collection of specimens for this study. This research was carried out in compliance with the relevant laws of China.

\section{Consent for publication}

Not applicable.

\section{Competing interests}

The authors declare that they have no competing interest.

\section{Author details}

${ }^{1}$ The Provincial and Ministerial co-founded collaborative innovation center for R \& D in Tibet characteristic Agricultural and Animal Husbandry resources, Tibet Agriculture and Animal Husbandry University, Nyingchi of Tibet 860000 , China

${ }^{2}$ Key Laboratory of Forest Ecology in Tibet Plateau, Tibet Agricultural \& Animal Husbandry University, Ministry of Education, Nyingchi, Tibet 860000 , China

${ }^{3}$ The Center for Xizang Chinese (Tibetan) Medicine Resource, Tibet Agriculture and Animal Husbandry University, Nyingchi of Tibet 860000 , China

${ }^{4}$ Germplasm Bank of Wild Species, Kunming Institute of Botany, Chinese Academy of Sciences, Kunming, Yunnan 650201, China

${ }^{5}$ The Provincial and Ministerial co-founded collaborative innovation center for R \& D in Tibet characteristic Agricultural and Animal Husbandry resources, School of Life Sciences, Southwest University, Chongqing 400715, China

\section{References}

1. Fu SX, Fu KJ: The Flora of China. Sci. Press 1984, 34(1):159.

2. Panossian A, Wikman G, Sarris J: Rosenroot (Rhodiola rosea): Traditional use, chemical composition, pharmacology and clinical efficacy. Phytomedicine 2010, 17(7):481-493. 
3. Mayuzumi S, Ohba H: The Phylogenetic Position of Eastern Asian Sedoideae (Crassulaceae) Inferred from Chloroplast and Nuclear DNA Sequences. Systematic Botany 2004, 29(3): 587-598.

4. Zhao D-N, Ren Y, Zhang J-Q: Conservation and innovation: Plastome evolution during rapid radiation of Rhodiola on the Qinghai-Tibetan Plateau. Molecular Phylogenetics and Evolution 2020, 144:106713.

5. Hong DX, Su JS, Wen J, Zhang J, Zhang Y: [Resource investigation about Tibetan medicine Rhodiola kirilowii]. Zhongguo Zhong Yao Za Zhi 2017, 42(6):1202-1206.

6. Qiang W, Xiao R, Lan F, Yan Q: Study on Stage, Questions and Strategies of Resource Plant Rhodiola L. Journal of Xinjiang Agricultural University 2002.

7. $\mathrm{Li} \mathrm{T}$, Zhang $\mathrm{H}$ : Application of microscopy in authentication of traditional Tibetan medicinal plants of five Rhodiola (Crassulaceae) alpine species by comparative anatomy and micromorphology. Microscopy Research \& Technique 2010, 71(6):448-458.

8. Xin T, Li X, Yao H, Lin Y, Ma X, Cheng R, Song J, Ni L, Fan C, Chen S: Survey of commercial Rhodiola products revealed species diversity and potential safety issues. Scientific Reports 2015, 5(8337): DOI: 10.1038/srep08337.

9. Wang ML, Ren XL, Cui JL, Wang JH: Genetic diversity of wild plants in Rhodiola L. with two molecular marker methods of RAPD and ISSR. 2016, 47:469-473.

10. Li T, Su C, Li LX, Li C, Si MX: Identification of Rhodiola quadrifida and Rhodiola crenulata based on NMR fingerprint and chemical pattern recognition method. Chinese Traditional and Herbal Drugs 2018, 49:3918-3925.

11. Zhu R-W, Li Y-C, Zhong D-L, Zhang J-Q: Establishment of the most comprehensive ITS2 barcode database to date of the traditional medicinal plant Rhodiola (Crassulaceae). Scientific Reports 2017, 7(1):10051.

12. Booker A, Zhai L, Gkouva C, Li S, Heinrich M: From Traditional Resource to Global Commodities:-A Comparison of Rhodiola Species Using NMR Spectroscopy-Metabolomics and HPTLC. In: Frontiers in pharmacology. 2016, 7:254. Doi: 10.3389/fphar.2016.00254.

13. Hebert PDN, Gregory TR: The Promise of DNA Barcoding for Taxonomy. Systematic Biology 2005, 54(5):852-859.

14. Jansen RK, Zhengqiu C, Raubeson LA, Henry D, Depamphilis CW, James LM, Kai F, Müller, Mary GB, Haberle RC, Hansen AK: Analysis of $\mathbf{8 1}$ genes from 64 plastid genomes resolves relationships in angiosperms and identifies genome-scale evolutionary patterns. Proceedings of the National Academy of Sciences of the United States of America 2007, 104(49):19369-19374.

15. Chen X, Zhou J, Cui Y, Wang Y, Duan B, Yao H: Identification of Ligularia Herbs Using the Complete Chloroplast Genome as a Super-Barcode. In: Frontiers in pharmacology. vol. 9; 2018: 695.

16. Zhong ZM: Study on identification and systematic classification of Dendrobium with DNA barcode. CNKI.

17. Bull, N. L: Compound Microsatellite Repeats: Practical and Theoretical Features. Genome Research 1999, 9(9):830-838. 
18. Zhao K, Li L, Lu Y, Yang J, Zhang Z, Zhao F, Quan H, Ma X, Liao Z, Lan X: Characterization and Comparative Analysis of Two Rheum Complete Chloroplast Genomes. BioMed Research International 2020, 2020(4):1-11.

19. Kuang DY, Wu H, Wang YL, Gao LM, Zhang SZ, Lu L: Complete chloroplast genome sequence of Magnolia kwangsiensis (Magnoliaceae): implication for DNA barcoding and population genetics. Genome 2011, 54(8):663-673.

20. Wang W, Yu H, Wang J, Lei W, Gao J, Qiu X, Wang J: The Complete Chloroplast Genome Sequences of the Medicinal Plant Forsythia suspensa (Oleaceae). International Journal of Molecular Sciences 2017, 18(11):2288.

21. Kim S-H, Yang J, Park J, Yamada T, Maki M, Kim S-C: Comparison of Whole Plastome Sequences between Thermogenic Skunk Cabbage Symplocarpus renifolius and Nonthermogenic S. nipponicus (Orontioideae; Araceae) in East Asia. International journal of molecular sciences 2019, 20(19):4678.

22. Sajjad A, L. KA, R. KA, Muhammad W, Sang-Mo K, A. KM, Seok-Min L, In-Jung L: Complete Chloroplast Genome of Nicotiana otophora and its Comparison with Related Species. Frontiers in Plant Science, 7. Doi: 10.3389/fpls.2016.00843.

23. Bremer B, Bremer K, Chase MWF, Michael F, Reveal JL, Soltis DE, Soltis PS, Stevens PF, Anderberg AA, Moore MJ, Olmstead RG: An update of the Angiosperm Phylogeny Group classification for the orders and families of flowering plants: APG III. Botanical Journal of the Linnean Society 2009, 161(2):105121.

24. Allen GC, Floresvergara MA, Krasynanski S, Kumar S, Thompson WF: A modified protocol for rapid DNA isolation from plant tissues using cetyltrimethylammonium bromide. Nature Protocols 2006, 1(5):2320.

25. Patel RK, Jain M: NGS QC Toolkit: A Platform for Quality Control of Next-Generation Sequencing Data. Springer 2015: 544-548.

26. Nicolas D, Patrick M, Guillaume S: NOVOPlasty: de novo assembly of organelle genomes from whole genome data. Nucleic Acids Research 2017(4): 45:e18.Doi: 10.1093/nar/gkw955.

27. Tillich M, Lehwark P, Pellizzer T, Ulbricht-Jones ES, Fischer A, Bock R, Greiner S: GeSeq-versatile and accurate annotation of organelle genomes. Nucleic acids research 2017, 45(1):6-11.

28. Matthew K, Richard M, Amy W, Steven SH, Matthew C, Shane S, Simon B, Alex C, Sidney M, Chris D: Geneious Basic: an integrated and extendable desktop software platform for the organization and analysis of sequence data. Bioinformatics 2012, 28(12):1647-1649.

29. Brooks A, Lowe T: The tRNAscan-SE, snoscan and snoGPS web servers for the detection of tRNAs and snoRNAs. Nucleic acids research 2005, 33:686-689.

30. Marc L, Oliver D, Sabine K, Ralph B: OrganellarGenomeDRAW-a suite of tools for generating physical maps of plastid and mitochondrial genomes and visualizing expression data sets. Nucleic Acids Research 2013, 41(Web Server issue):575.

31. Frazer KA, Lior P, Alexander P, Rubin EM, Inna D: VISTA: computational tools for comparative genomics. Nucleic Acids Research 2004, 32(Web Server issue): 273-279.. 
32. Rozas J, Ferrer-Mata A, Sánchez-DelBarrio JC, Guirao-Rico S, Librado P, Ramos-Onsins SE, SánchezGracia A: DnaSP 6: DNA sequence polymorphism analysis of large data sets. Molecular biology and evolution 2017, 34(12):3299-3302.

33. Kurtz S, Choudhuri JV, Ohlebusch E, Schleiermacher C, Stoye J, Giegerich R: REPuter: the manifold applications of repeat analysis on a genomic scale. Nucleic Acids Research 2001, 29(22):46334642.

34. Thiel T,., Michalek W, ., Varshney RK, Graner A,. Exploiting EST databases for the development and characterization of gene-derived SSR-markers in barley (Hordeum vulgare L.). Theoretical \& Applied Genetics 2003, 106(3):411-422.

35. Katoh K, Standley DM: MAFFT Multiple Sequence Alignment Software Version 7: Improvements in Performance and Usability. Molecular Biology and Evolution 2013, 30(4):772-780.

36. Ronquist F, Teslenko M, Der Mark PV, Ayres DL, Darling AE, Hohna S, Larget B, Liu L, Suchard MA, Huelsenbeck JP: MrBayes 3.2: Efficient Bayesian Phylogenetic Inference and Model Choice across a Large Model Space. Systematic Biology 2012, 61(3):539-542.

37. Millen RS, Olmstead RG, Adams KL, Palmer JD, Lao NT, Heggie L, Kavanagh TA, Hibberd JM, Gray JC, Morden CW: Many Parallel Losses of infA from Chloroplast DNA during Angiosperm Evolution with Multiple Independent Transfers to the Nucleus. The Plant Cel/ 2001, 13(3):645-658.

38. Nock CJ, Waters DL, Edwards MA, Bowen SG, Rice N, Cordeiro GM, Henry RJ: Chloroplast genome sequences from total DNA for plant identification. Plant biotechnology journal 2011, 9(3):328-333.

39. Zhao K, Li L, Quan H, Yang J, Lan X: Comparative Analyses of Chloroplast Genomes From 14 Zanthoxylum Species: Identification of Variable DNA Markers and Phylogenetic Relationships Within the Genus. Frontiers in Plant Science 2021, 11:605793. Doi:10.3389/fpls.2020.605793.

40. Jansen RK, Cai Z, Raubeson LA, Daniell H, Boore JL: Analysis of 81 genes from 64 plastid genomes resolves relationships in angiosperms and identifies genome-scale evolutionary patterns. Proceedings of the National Academy of Sciences 2008, 104(49):19369-19374.

41. Xue S, Shi T, Luo W, Ni X, Iqbal S, Ni Z, Huang X, Yao D, Shen Z, Gao Z: Comparative analysis of the complete chloroplast genome among Prunus mume,P.armeniaca,and P.salicina. Horticulture Research 2019, 6(1):13.

42. Luo X, Tinker NA, Xing F, Zhang H, Sha L, Kang H, Ding C, Jing L, Li Z, Yang R: Phylogeny and maternal donor of Kengyilia species (Poaceae: Triticeae) based on three cpDNA (matK, rbcL and trnH-psbA) sequences. Biochemical Systematics \& Ecology 2012, 44:61-69.

43. Cabelin V, Alejandro G: Efficiency of matK, rbcL, trnH-psbA, and trnL-F (cpDNA) to Molecularly Authenticate Philippine Ethnomedicinal Apocynaceae Through DNA Barcoding. Pharmacogn Mag 2016, 12(46):S384-388.

44. Jin WT, Schuiteman A, Chase MW, Li JW, Chung SW, Hsu TC, Jin XH: Phylogenetics of subtribe Orchidinae s.I. (Orchidaceae; Orchidoideae) based on seven markers (plastid matK, psaB, rbcL, trnL-F, trnH-psba, and nuclear nrITS, Xdh): implications for generic delimitation. Bmc Plant Biology 2017, 17(1):222. 
45. Olsson S, Grivet D, Cidvian J: Species-diagnostic markers in the genus Pinus: evaluation of the chloroplast regions matK and ycf1. 2018,27(3): e016.

46. Mayuzumi S, Ohba H: The phylogenetic position of eastern Asian Sedoideae (Crassulaceae) inferred from chloroplast and nuclear DNA sequences. Systematic botany 2004, 29(3):587-598.

47. Hermsmeier U, Grann J, Plescher A: Rhodiola integrifolia: hybrid origin and Asian relatives. Botanybotanique 2012, 90(11):1186-1190.

48. Bremer B, Bremer K, Chase M, Reveal J, Zmarzty S: An update of the Angiosperm Phylogeny Group classification for the orders and families of flowering plants: APG II. Botanical Journal of the Linnean Society 2003, 141(4):399-436.

\section{Figures}

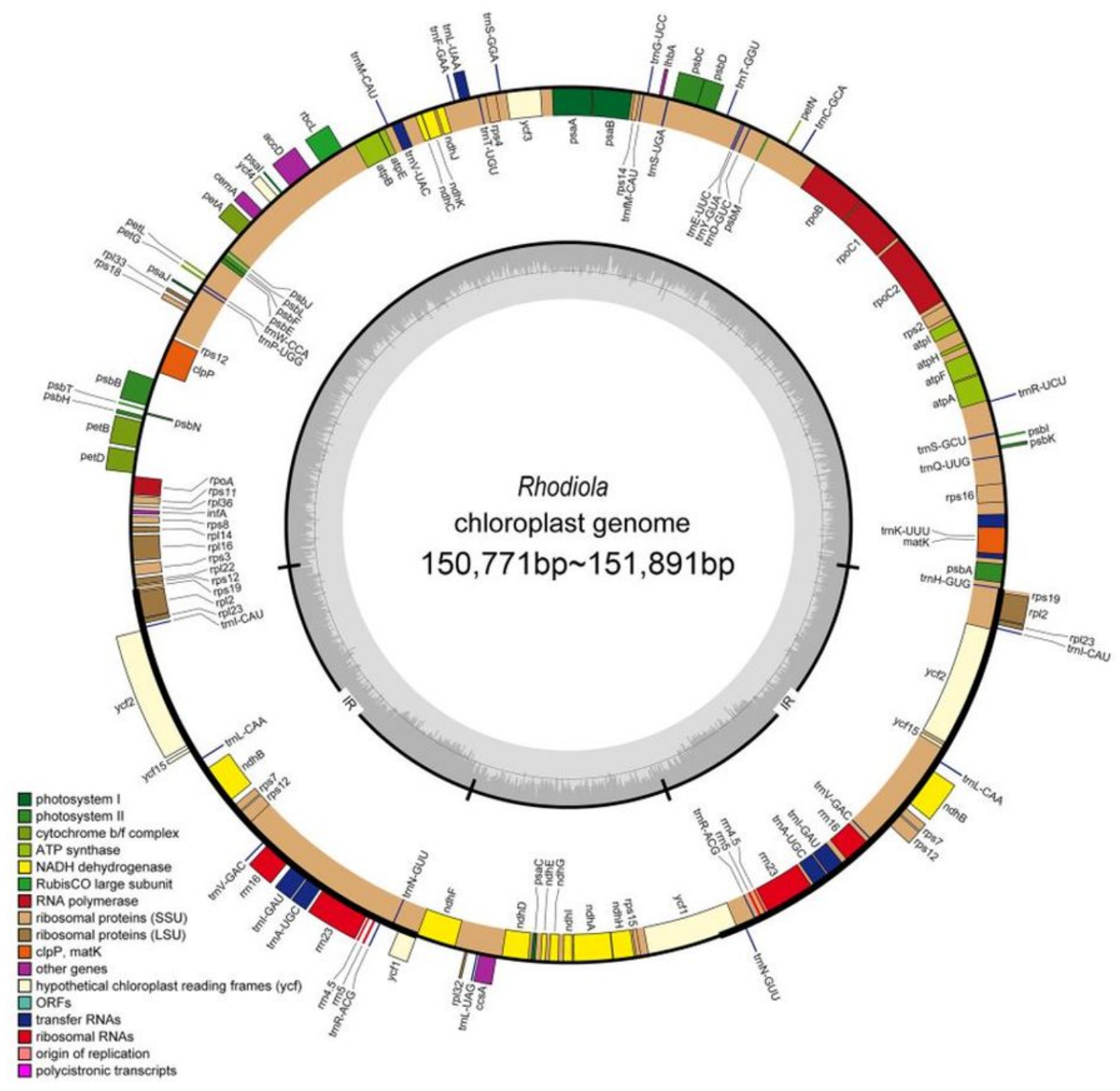

Figure 1 
Gene maps of the complete cp genome of six species of Rhodiola. Genes drawn outside of the map circle are transcribed clockwise, while those drawn inside are transcribed counter-clockwise. The darker gray in the inner circle corresponds to GC content while the lighter gray corresponds to AT content.
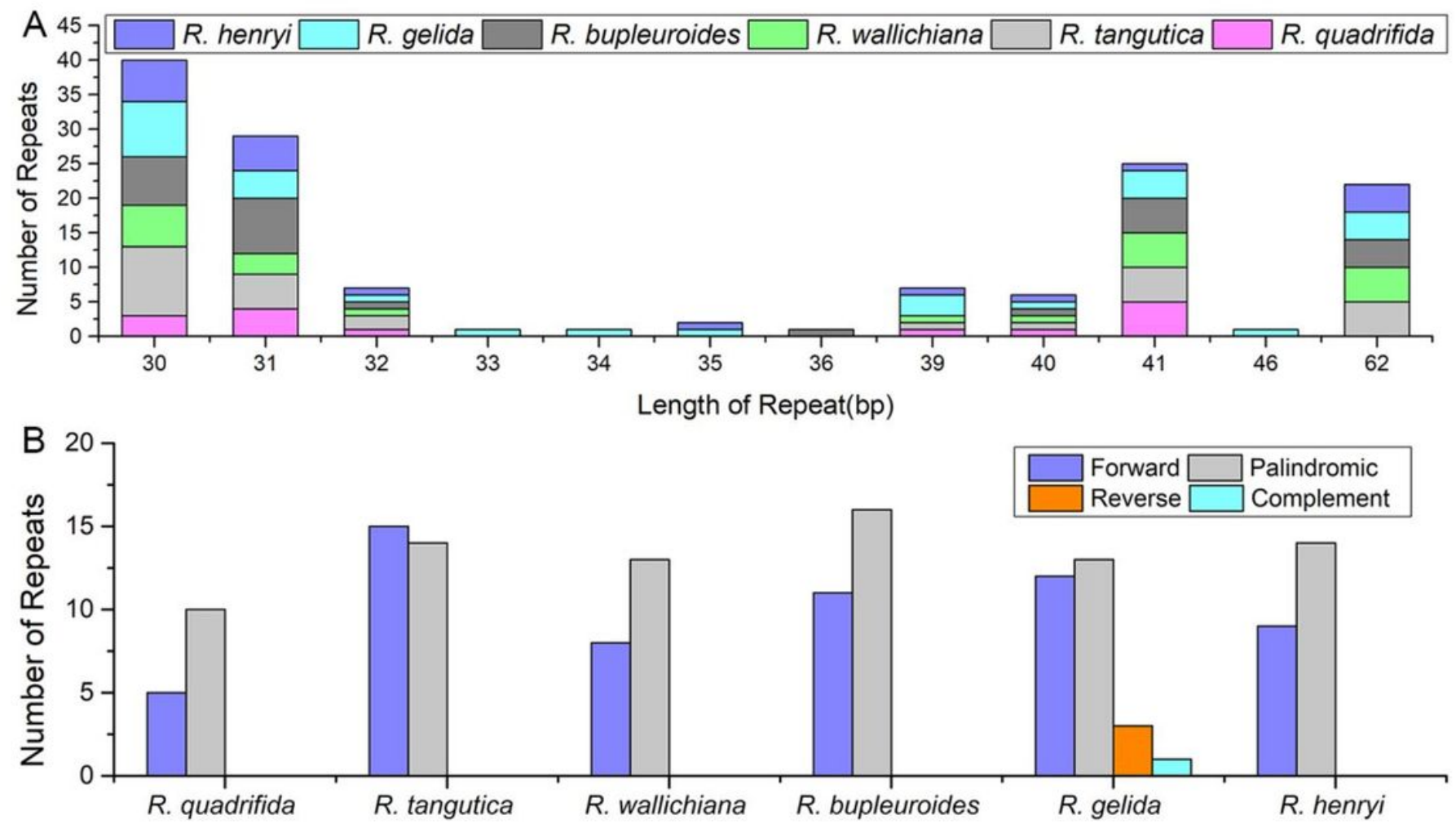

\section{Figure 2}

The number of long repeats in the whole cp genome sequence of the 6 Rhodiola species. (A) Frequency of repeats more than $30 \mathrm{bp}$ long. (B) Frequency of repeat type. 


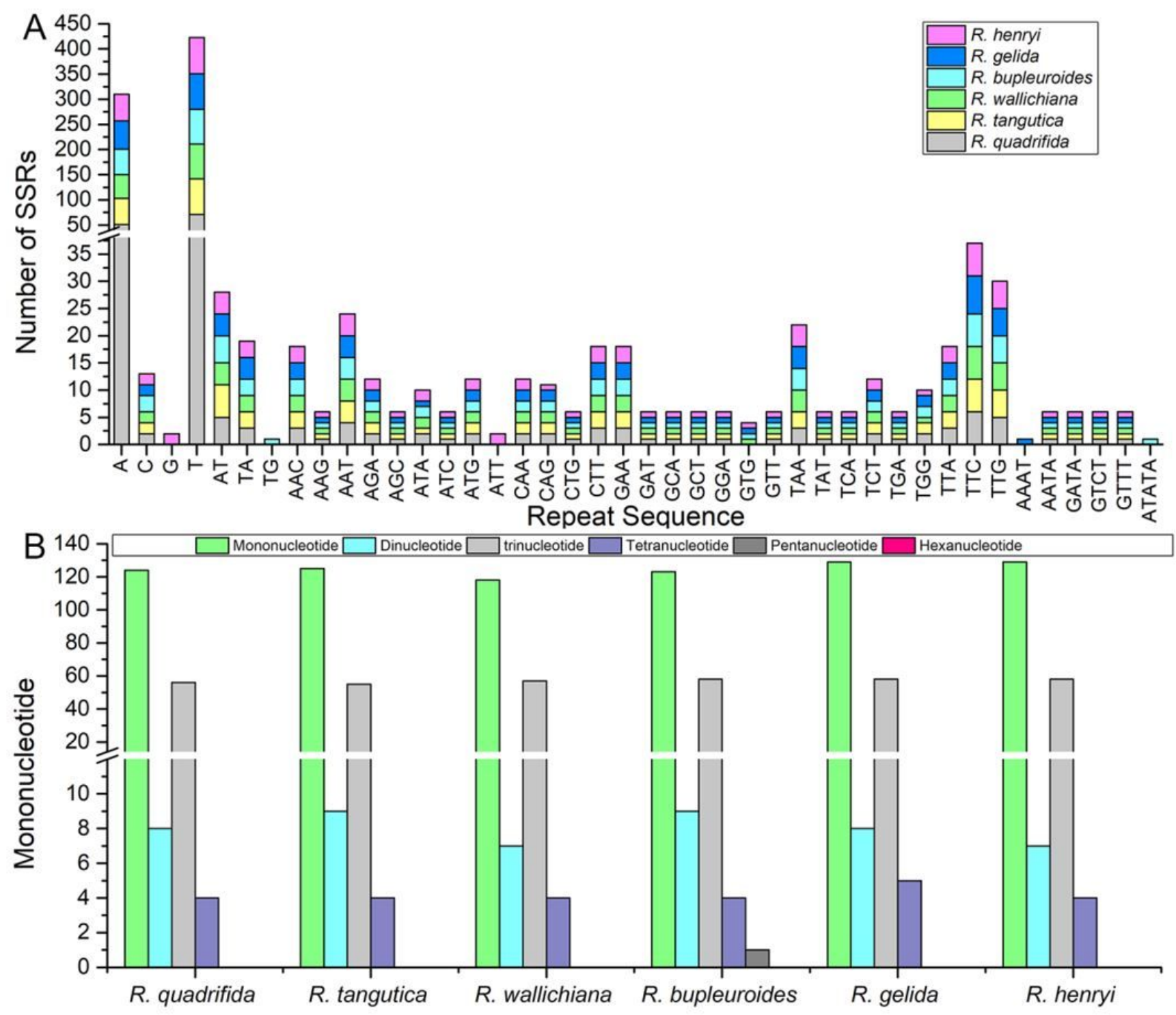

Figure 3

Analysis of SSRs in the 6 Rhodiola cp genomes. (A)Frequency of common motifs in the 6 Rhodiola cp genomes. (B)Number of different SSR types detected in the 6 Rhodiola cp genomes. 

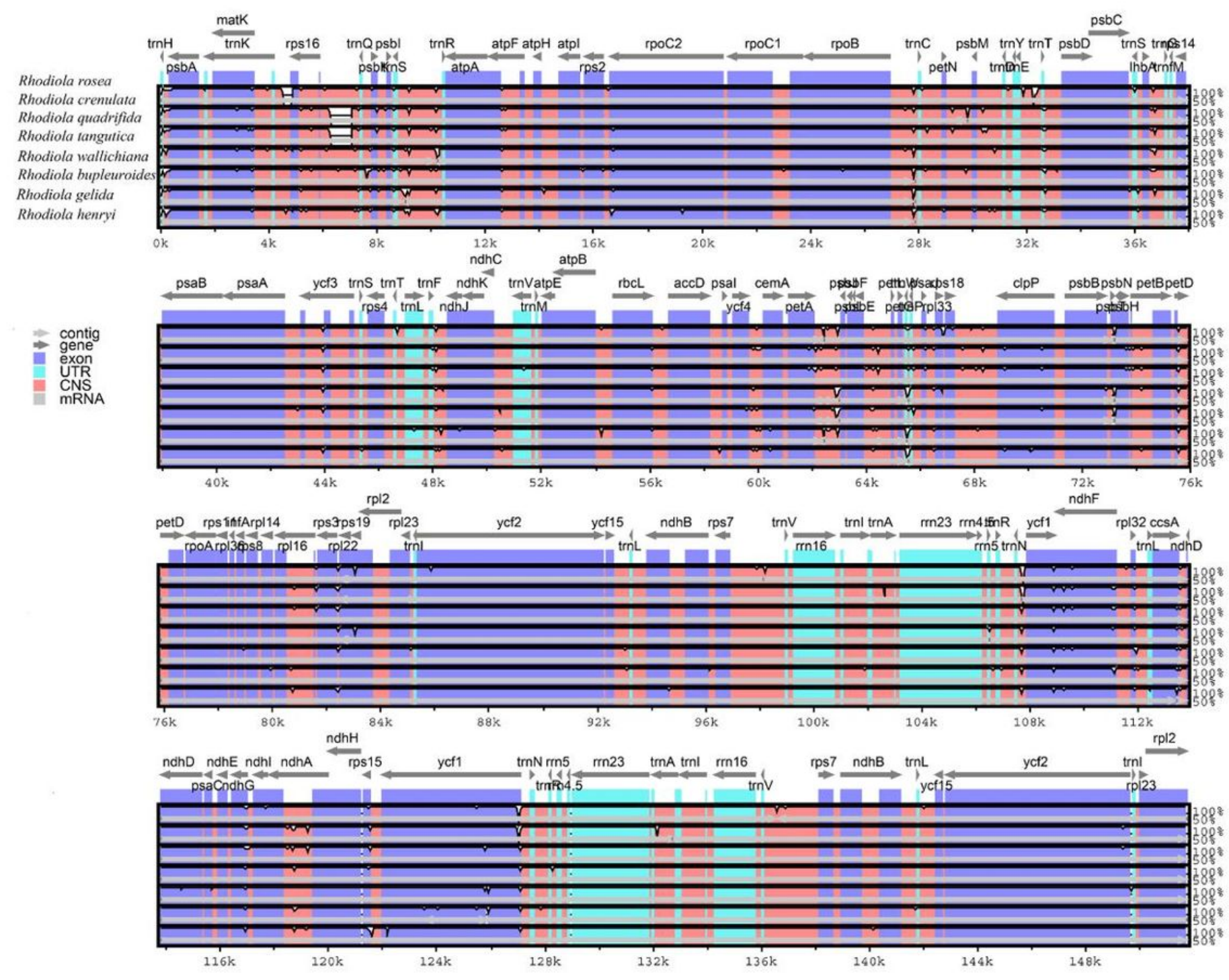

\section{Figure 4}

Sequence alignment of chloroplast genomes of eight Rhodiola species. Sequence identity plot comparing the chloroplast genomes with $R$. rosea as a reference using mVISTA. The grey arrows and thick black lines above the alignment indicate genes with their orientation. The $Y$-axis represents the identity from $50 \%$ to $100 \%$. 


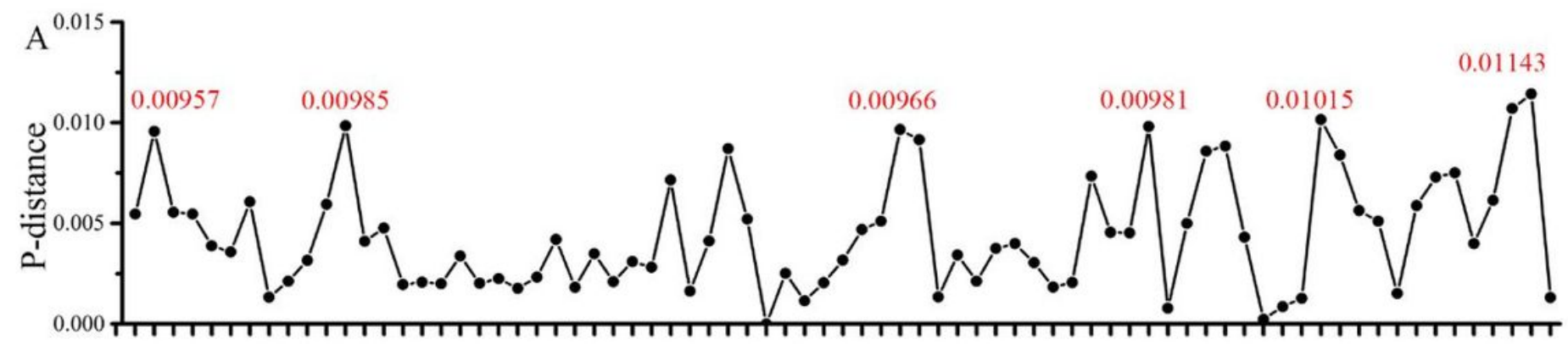
Pans

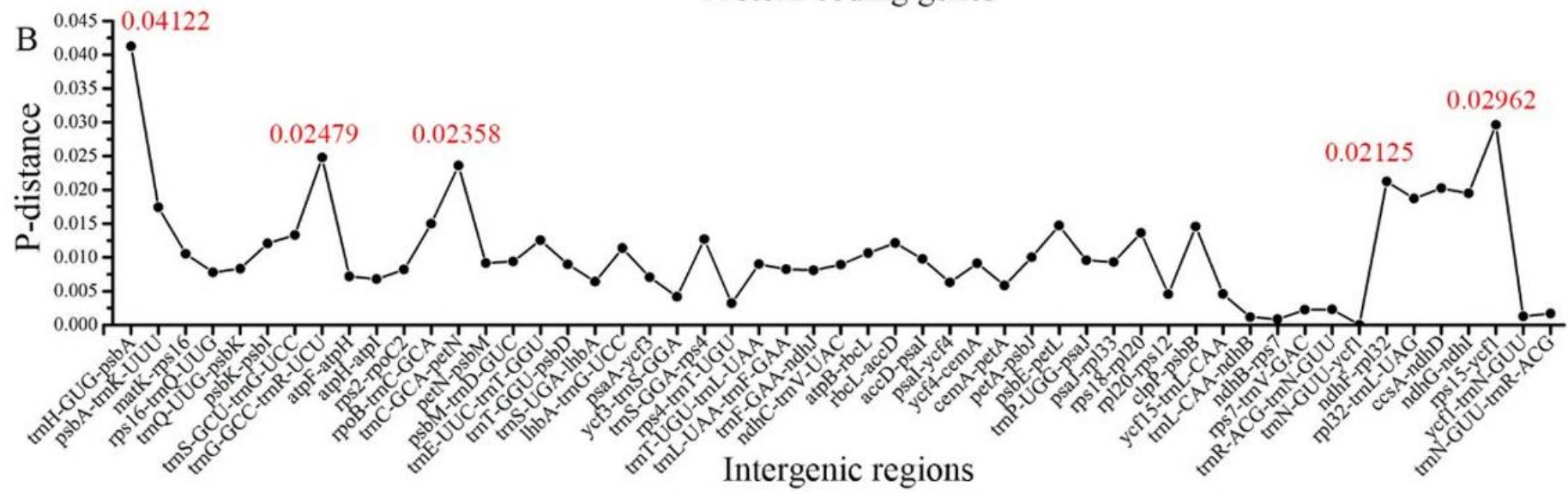

Protein-coding genes

Figure 5

The nucleotide variability (Pi) values were compared among 22 Rhodiola species. (A) The P-distance value of protein-coding genes. (B) The P-distance value of intergenic regions. 


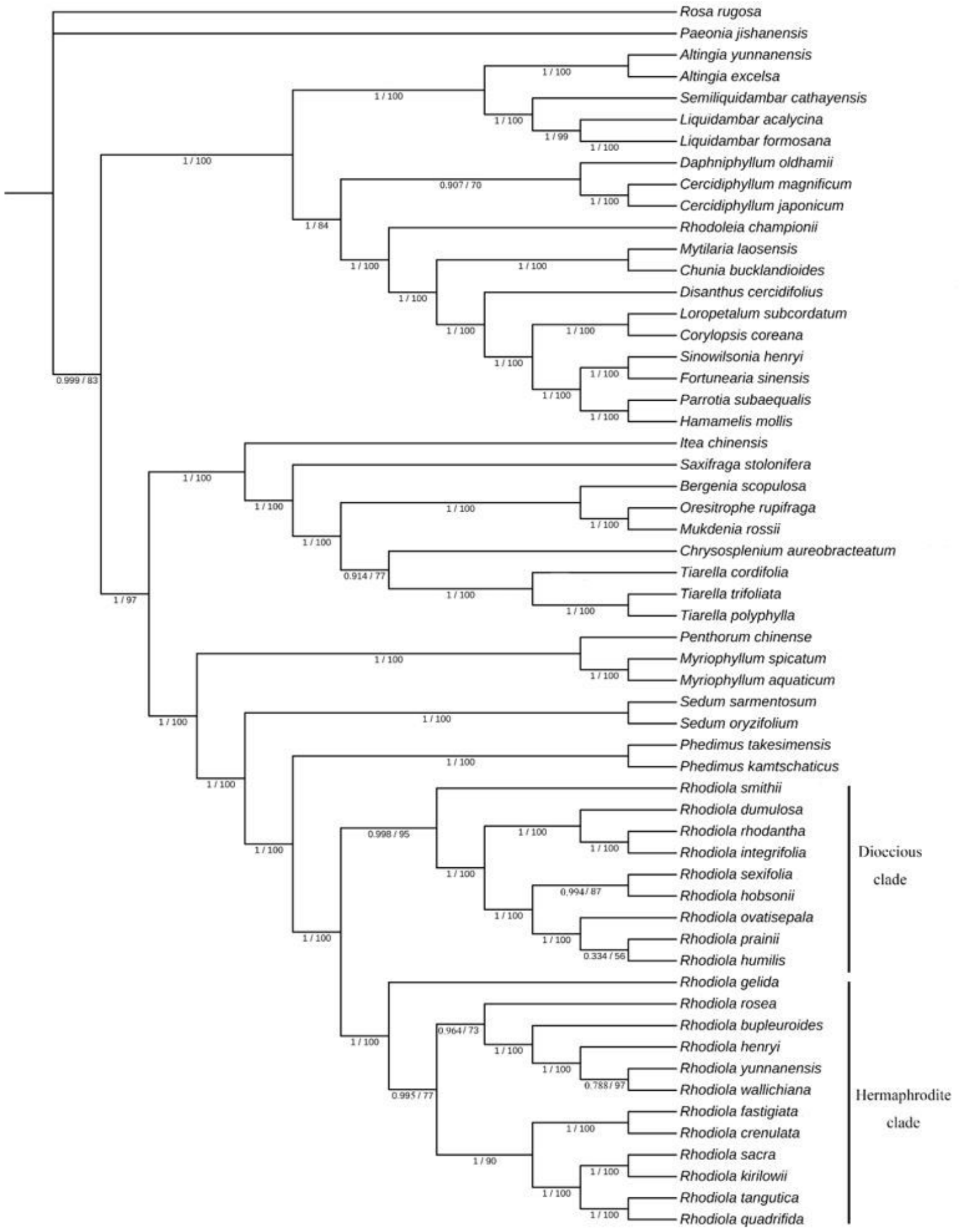

\section{Figure 6}

Phylogenetic tree based on 38 protein-coding genes of 55 available complete $\mathrm{cp}$ genome sequences in Saxifragales. The tree was generated using a ML method with 1000 bootstrap replicates. Numbers on the nodes indicate bootstrap values. 


\section{Supplementary Files}

This is a list of supplementary files associated with this preprint. Click to download.

- TableS1.SummaryoftheRhodiolachloroplastgenomecharacteristics.xlsx

- TableS2.ListofgenesinsixRhodiolachloroplastgenomes..docx

- TableS3.Listofspeciesusedtoevaluatethesequencedivergence.docx

- TableS4.Listofspeciesusedforphylogenetictreeconstruction.docx

- TableS5.Listof38proteincodinggenesusedforphylogenetictreeconstruction..docx

- GBS6.DataforournewlyassembledsixRhodiolachloroplastgenomes.zip 